\title{
Methemoglobin and hemolytic anemia as potential markers for drug side-effects
}

\author{
H. Singh \& E. T. Purnell \\ Department of Natural Sciences and Mathematics, \\ College of Sciences and Technology, Savannah State University, \\ Savannah, USA
}

\begin{abstract}
Methemoglobinemia and hemolytic anemia are the most prominent side-effects of a wide variety of arylamine drugs including agricultural and industrial chemicals. Symptoms of this hemotoxicity include headache, fatigue, dizziness respiratory and cardiac arrest, and possibly death. Parent compounds are usually converted to their toxic metabolites (N-hydroxylamine) and react with oxyhemoglobin with the consequent reduction of molecular oxygen to active oxygen species leading to hemotoxic damage. We are investigating the role of redox cycling and an alternative hypothesis; viz., that a "hydroxylaminecentered" radical formed during an arylhydroxylamine-oxyhemoglobin reaction causes hemotoxic damage. We examined the time-course methemoglobin potential and hemolytic anemia potential of four laboratory synthesized halogenated phenylhydroxylamines based on their decreasing electronegativity: phenylhydroxylamine (PHA), p-fluoro-, p-bromo-, and p-iodo-PHA. Methemoglobin (MetHb) was determined spectrophotometrically and erythrocyte hemolysis was studied by collecting whole blood from male Sprague-Dawley rats, labeling the cells with radioactive chromium-51 and by infusing the labeled erythrocytes via tail vein in isologous rats. The time course of blood radioactivity was followed by serial sampling of blood from the orbital sinus for 14 days. Results showed dose- and time-dependent changes in the induction of methemoglobin by aniline-derivatives. The MetHb levels peaked to more than 70 percent within 10 minutes at $60 \mu \mathrm{M}$ and remained elevated for 240 minutes in certain treatments. All tested agents produced dose-dependent reductions in the labeled red blood cells indicating the loss of blood cells from circulation. The dose- and time-dependent methemoglobin and hemolytic anemia responses suggest these hydroxylamines as potential active metabolites and biomarkers that may mediate aniline-induced hemotoxicity. The minimum dose required to induce these effects varies with the test agent based on their electronegativity potential.
\end{abstract}

Keywords: aniline, methemoglobin, hemolytic anemia, hemoglobin biomarkers, halogenated phenylhyroxylamines. 


\section{Introduction}

Biological markers are usually endpoints in events leading from exposures to toxic damage. Such markers are potentially useful for risk assessments by linking exposures to given amounts of a chemical with the induction of specific health effects. Strategies for the use of biomarkers in the prediction of risk for disease should include mechanistic research to determine the earliest steps leading to disease. Studies should be done to determine which of the DNA and hemoglobin adducts formed by a chemical may lead to neoplasia based on chemical and physical properties of a toxicant. Once the mechanism of disease is known, pharmacodynamic models of the disease process may help in using biomarkers to predict risk and develop safer drugs. In general, DNA and hemoglobin adducts formed by a chemical exposure are likely to lead to a toxic injury based on structural properties. Therefore, it is desirable to understand the relationship between exposures and the probability of disease development in relation to structure and physical properties of a chemical (Henderson [1]).

In this study, we present mechanistic data on the relationship between the chemical structure and its two potential blood biomarkers, methemoglobin and blood hemolysis as toxic side-effects accompanied after treatment with industrial chemical aniline and its derivatives. Studies in rats with aniline and dapsone have led to the identification of N-hydroxy metabolites as the potentials hemotoxic mediators for certain chemicals especially arylamine compounds (Harrison and Jollow [2, 3] Grossman et al. [4]). There is little information, however, regarding the potential hemotoxicity from exposure to halogenated arylamine derivatives, particularly industrial and agricultural chemicals such as aniline.

Methemoglobin (MetHb) is formed in erythrocytes even under normal conditions but reducing mechanisms present in the normal cell maintain most of the hemoglobin in a functioning state. The normal reducing mechanism utilizes NADH (reduced nicotine adenine dinucleotide) methemoglobin reductase and NADPH that provide electrons and protons for the enzymatic reduction of methemoglobin. In most cases, the methemoglobinemia is an acquired condition and results from the action of certain drugs, chemical agents and food. Certain nitro- and amino-derivatives of the aromatic compounds like aniline, nitrobenzene, nitrotoluene, phenylhydromine, and sulfonamides have been known to cause methemoglobinemia (Kiese [5]).

A number of toxic metabolites that produce methemoglobin are also responsible for inducing hemolytic activity. Compounds of industrial interest (aniline), agricultural chemicals (Propanil) and drugs (dapsone, phenacetin, primaquine) have been shown as methemoglobinemic and hemolytic agents. The objective of this study was to examine the methemoglobinemic and hemolytic capacities of structurally related halogenated arylhydroxylamines with decreasing electronegativity such as N-hydroxy derivatives of aniline phenylhydroxylamine: p-fluoro-, p-bromo-, and p-iodo-phenylhydroxylamines.

Aniline (benzeamine, phenylamine, MW 93.12) is an oily liquid, colorless but darkens upon exposure to light and air. This chemical is used as a solvent in manufacturing dyes, medicinal products, resins, perfumes and shoe polish. 
The present study attempts to understand the mechanisms underlying arylamine-induced methemoglobinemia and hemolytic anemia biomarkers with respect to structure/activity requirements of aniline derivative-analogs.

\section{Materials and methods}

\subsection{Synthesis of arylhydroxylamines}

Various halogenated hydroxylamines were synthesized from the corresponding nitroanilines by zinc dust $/ \mathrm{NH}_{4} \mathrm{CI}$ reduction or by catalytic reduction with hydrogen (Harrison and Jollow [2], Grossman and Jollow [4]). Both techniques have been used routinely for the preparation of arylhydroxylamines in the literature. The following synthesized arylhydroxylamines were crystallized purified and stored at $-80^{\circ} \mathrm{C}$ : phenylhydroxylamine (PHA), 4-fluoro-, 4-bromo-, and 4-iodo-phenylhydroxylamines. Structures of these analogs were confirmed by MS, NMR and HPLC.

\subsection{Blood collection}

Whole blood was collected from male, Sprague-Dawley rats, weighing 100-150 gms via aortic puncture. Seven to $10 \mathrm{ml}$ of whole blood was collected into heparinized syringes and placed in $50 \mathrm{ml}$ conical tubes. The blood was washed at $37^{0} \mathrm{C}$ with PBSG at pH 7.4 then centrifuged at $10,000 \mathrm{rpm}$ for 5 minutes in a desktop Eppendorf Centrifuge 5403. The supernatant was aspirated into a vacuum flask. The resulting RBCs were washed twice more with the final wash lasting for 7 minutes to better pellet the cells. The final pellet was diluted with the phosphate buffered saline solution with glucose (PBSG) to yield a $40 \%$ hematocrit.

\subsection{Methemoglobin assay}

The halogenated phenylhydroxylamines were prepared in acetone to yield concentrations from $30-300 \mu \mathrm{M}$. Ten $\mu \mathrm{l}$ of the test agent versus the vehicle (acetone) was added to the PBSG. Two $\mathrm{ml}$ samples in scintillation vials for incubations were placed in a 37-degree shaker bath and methemoglobin levels were measured at various time points ending at 180-240 minutes. Fifteen ml plastic tubes, one for each sample, were placed on ice. Five $\mathrm{ml}$ of ice-cold hemolysis buffer (0.277\% Potassium Phosphate, 0.289\% Sodium Phosphate, and $0.05 \%$ Triton $\times 100$ ) was placed in each tube. Then $75 \mu$ of the $40 \%$ hematocrit blood was pipetted into $5 \mathrm{ml}$ of the hemolysis buffer and vortexed. For each sample, 4 cuvettes were arranged. To cuvettes 2 and 4, $20 \mu 1$ of $10 \% \mathrm{KCN}$ was added. To cuvettes 3 and 4, $20 \mu \mathrm{l}$ of $20 \% \mathrm{~K} 3 \mathrm{Fe}(\mathrm{CN})_{6}$ was added. One $\mathrm{ml}$ of the hemolyzed blood sample was pipetted into each of the 4 cuvettes. The absorbance was measured at $635 \mathrm{~nm}$ on an UltroSpec $2000 \mathrm{UV} / \mathrm{Visible}$ Spectrophotometer. Incubations in the presence and absence of the various chemical agents described above were performed in triplicate. Methemoglobin 
levels relative to total hemoglobin were measured by a modification of the spectrophotometric technique of Evelyn and Malloy [6].

\subsection{Measurement of the hemolytic response}

Rat erythrocytes were collected, washed three times with PBSG, labeled with radioactive sodium chromate $\left(0.1 \mathrm{~m}{ }^{51} \mathrm{Cr} / 1.0 \mathrm{ml}\right.$ of packed cells $)$, incubated for 15 minutes at $37^{0} \mathrm{C}$ on shaker bath, washed once again with PBSG, adjusted hematocrit to $40 \%$, treated cells with specified doses, incubated again for two hours at $37^{0} \mathrm{C}$ before injecting into rats $(0.5 \mathrm{ml} /$ animal $)$. The initial blood sample $75-\mu \mathrm{l}\left(\mathrm{T}_{0}\right)$ was taken from the orbital sinus within 30 minutes after the infusion of tagged erythrocytes in rats. Control incubations received acetone as a vehicle. The treated erythrocytes were administered via tail vein to isologous rats as previously described elsewhere (International Committee for Standardization in Hematology [7], Harrison and Jollow [2]). Serial blood samples (75 $\mu \mathrm{l}$ ) from each rat were then collected into heparinized capillary tubes at designated intervals for 14 days. At the end of experiment, all of the samples were counted in a well-type $\gamma$-counter, with the counts per minute above background for each sample expressed as a percentage of the $\mathrm{T}_{0}$ sample. Data were expressed in terms of $\%$ decrease in values relative to the radioactive ${ }^{51} \mathrm{Cr}$ values of the red cells in that animal at time zero $\left(\mathrm{T}_{0}\right)$ in the control.

\subsection{Animals}

Male Sprague Dawley rats weighing 100-150 gms were used for this study. The usage of rats falls into two categories: (1) RBC donors, (2) hemotoxicity assays. For RBC donation, the animals were placed under ether anesthesia and blood was obtained by cardiac puncture. For the hemotoxicity studies, the animals received radiolabelled $\mathrm{Cr}-51$ tagged red blood cells iv via tail vein under light ether anesthesia. Serial blood samples were taken subsequently from the orbital sinus. At the end of all experiments, the animals were placed under ether anesthesia and sacrificed by decapitation.

\section{Results}

\subsection{Methemoglobin}

Figure 1 shows dose dependent changes in the induction of methemoglobin following exposure to various halogenated phenylhydroxylamines. The MetHb induction peaked to $35 \%$ at $50 \mu \mathrm{M}, 56 \%$ at $100 \mu \mathrm{M}$ and to $67 \%$ at $150 \mu \mathrm{M}$ within 10 minutes before declining after treatment with para-fluoro-PHA. At the end of 240 minutes the, MetHb level was 5 to $42 \%$ depending on the dose compared to $2 \%$ in control. Similarly, para-bromo-PHA produced dose dependent MetHb that peaked to almost $78 \%$ within 20 minutes at $120 \mu \mathrm{M}$ before declining. The MetHb remained eight to thirteen-fold elevated (40 to 65 $\%$ ) compared to $5 \%$ in control at 240 minutes post-treatment. With para-iodoPHA, the MetHb level peaked to $75 \%$ at the $90 \mu \mathrm{M}$ dose before started 
declining. At the end of 240 minutes, the percent MetHb stayed elevated from 20 to $35 \%$ depending on the dose and this represented 3 to 7 fold increase in $\mathrm{MetHb}$ compared to control. The data presented showed the highest peak with para -bromo-PHA followed by para iodo-PHA, para -fluoro-PHA and PHA. With PHA, the MetHb peaked within 60 minutes before declining. However, with para -bromo- PHA and para -iodo-PHA, the percent MetHb remained elevated by 7-8 fold at the end of 240 minutes.
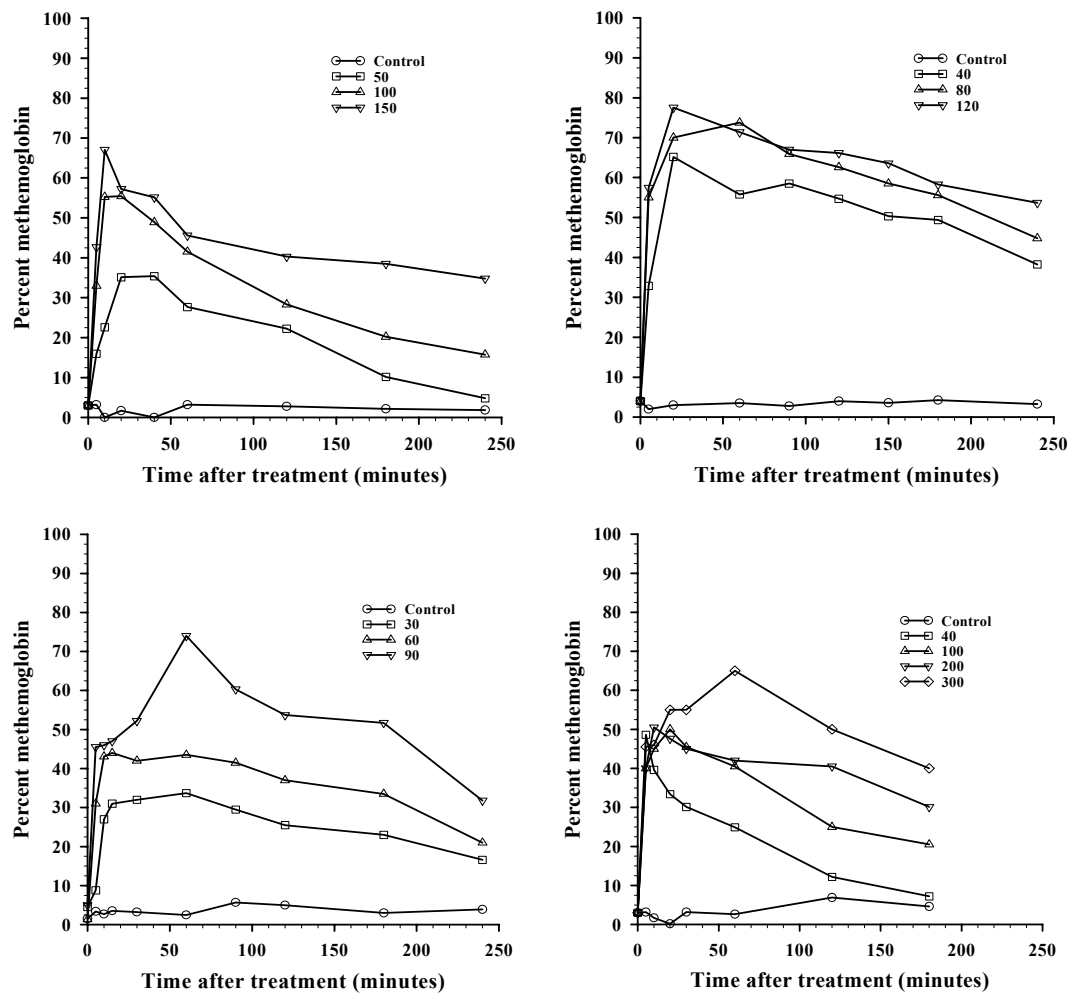

Figure 1: Time course of percent methemoglobin formation with para-fluoro-, para-bromo-, para-iodo-phenylhydroxylamine and PHA (dose in $\mu \mathrm{M})$.

\subsection{Hemolytic anemia}

The three aniline hydroxylamines tested produced dose dependent reduction in the labeled red blood cells indicating the loss of blood cells from circulation (Figure 2 a-c). The most pronounced reduction was observed at doses from 175 to $250 \mu \mathrm{M}$. The dose of $100 \mu \mathrm{M}$ appeared to be the threshold level and the dose of $325 \mu \mathrm{M}$ did not show much further reduction in the radioactivity than that 
observed at $250 \mu \mathrm{M}$. Results showed that para -iodo-PHA was two times more toxic than para-fluoro-PHA in the induction of hemolytic anemia after treatment with $175 \mu \mathrm{M}$. All the three tested halogenated phenylhydroxylamines appeared to be potential biomarkers in the induction of hemolytic anemia in this study.
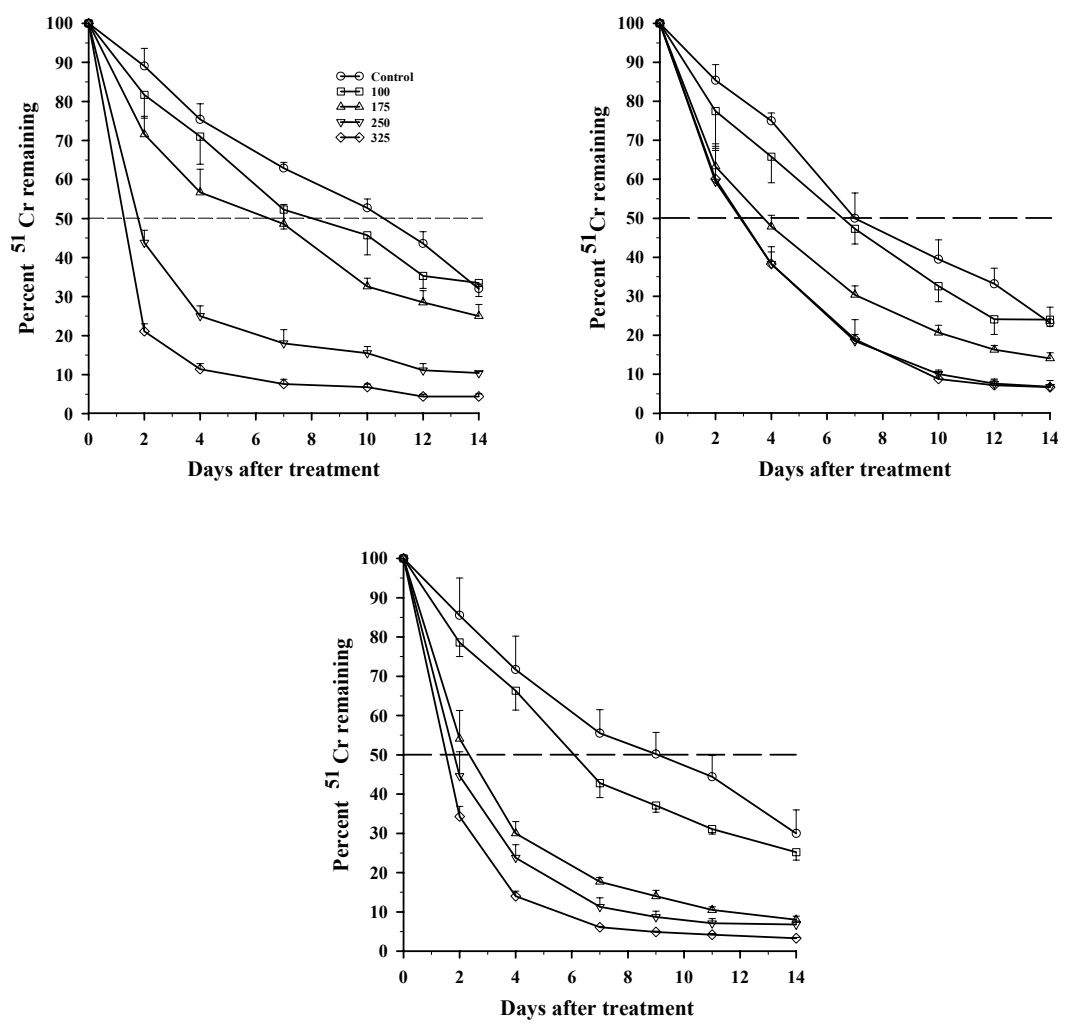

Figure 2: Percent ${ }^{51} \mathrm{Cr}$ remaining in erythrocytes after treatment with parafluoro-, para-bromo-, and para-iodo-PHA (dose in $\mu \mathrm{M}$ ).

The hemolytic effect of para-bromo- and para-iodo-PHA as shown in Figure 2 was almost similar. At $100 \mu \mathrm{M}$, there was little effect on the reduction of tagged cells from circulation with either phenylhydoxylamine compared to respective controls but the level of tagged cells dramatically reduced in circulation as the dose increased to $250 \mu \mathrm{M}$ or above. It appeared that the maximum drastic effect peaked at $250 \mu \mathrm{M}$ with either phenylhydroxylamine, thereby, reducing the circulation of tagged cells to less than 10 percent compared to approximately 30 percent in control.

The $\mathrm{T}_{50}(50 \%$ removal from circulation) values for PHA at 150 and $250 \mu \mathrm{M}$ were approximately 4.0 days and 8.5 and 1.75 days with para-fluoro-PHA, respectively, compared to 8.5 days in control. 


\section{Discussion and conclusion}

The current study showed that induction MetHb and blood hemolysis varied with dose, time and the electronegativity of halogenated phenylhydroxyamines tested. The para -fluoro-PHA produced the most rapid response by elevating the MetHb level from 2-5 percent in control to almost 70 percent within 10 minutes before declining. Whereas the MetHb steadily declined with time in all cases except with para -bromo-PHA that still showed 40-65 percent MetHb at the end of 240 minutes experimental period compared to less than 5 percent in control.

Aniline induced a dose-dependent methemoglobinemia in rats after intraperitoneal injection, but did not cause methemoglobin formation when it was present in erythrocyte suspensions in concentrations associated with in vivo methemoglobinemia. This finding is consistent with previous reports in the literature and with the conclusion that methemoglobinemia after aniline is mediated by one or more toxic metabolites formed during the hepatic clearance of aniline (Kiese [5]). Although phenylhydroxylamine, nitrosobenzene, or further breakdown products of aniline metabolism have not been demonstrated in urine (von Jagow et al. [8]) but Kiese [9] has shown that nitrosobenzene is present in blood after aniline administration. Production of nitrosobenzene and/or phenylhydroxylamine from aniline has also been observed in microsomal systems (Kiese [5]) and in the isolated perfused rat liver (Eyer et al. [10]).

The time course of methemoglobinemia in rats given aniline halogenated hydroxylamines is much different from that observed after treatment of the rats with the other phenylhydroxylamines. In general, halogenated aniline-induced a much extended duration of methemoglobinemia for a given peak level than did PHA as was observed in the present study. Whereas the metabolites produced parallel increases in peak and total methemoglobinemia with increasing dose, higher doses of aniline analogs were associated with a disproportionate increase in the duration and level of methemoglobinemia. This was confirmed in the present study.

Among the halogenated anilines the p-substituted compounds have been found to be most active in cats (McLean et al. [11]) and dogs (Kiese [12]). In dogs the high yield of ferrihemoglobin by 4-chloroaniline is due to the slow elimination of the parent amine and its N-hydroxy metabolite. According to Kiese and Renner [13], 4-Chlorophenylhydroxylamine and 4-chloronitrosobenzene accumulate to high concentrations, up to $2 \times 10^{-4} \mathrm{M}$ and probably produce most of the ferrihemoglobin. Ferrihemoglobin formation by halogen-anilines has also been observed in humans (Williams and Challis [14], Hughes and Treon [15], Faivre et al. [16]).

All three aniline hydroxylamines tested produced dose-dependent reduction in the labeled red blood cells indicating the loss of blood cells from circulation. The most pronounced reduction was observed at doses from 175 to $250 \mu \mathrm{M}$. The dose of $100 \mu \mathrm{M}$ appeared to be the threshold level and the dose of $325 \mu \mathrm{M}$ did not show much further reduction in the radioactivity than that observed at 250 $\mu \mathrm{M}$. Results showed that para -iodo-PHA was two times more toxic than para fluoro-PHA in the induction of hemolytic anemia in rats. These three 
phenylhydroxylamines tested appeared to be active metabolites that mediate aniline-induced hemolytic anemia. The study showed the following hemotoxic potencies of the hydroxylamines tested: p-iodo-PHA $>$ p-bromo-PHA $>$ p-fluoroPHA $>$ PHA $>$. Overall, aniline-derivatives produced a dose-dependent reduction in the circulation of tagged red cells as indicated by the radioactivity count.

It is generally known that hemolytic arylamines require metabolism to Nhydroxylamine's in order to produce toxic effects on red blood cells. The reactive N-hydroxy metabolites subsequently react with oxyhemoglobin to produce reactive oxygen species and sulfur-center free radical of hemoglobin resulting in formation of adducts with a variety of membrane associated proteins altering cytoskeletal structure and function. These membrane alterations result in the premature removal of damaged cells from the circulation by the spleen. This study was an attempt to compare the hemotoxicity of a variety of laboratorysynthesized arylhydroxylamine analogs of aniline selected based upon their differences in electronegativity. The aryl hydroxylamines selected for examination vary in electron donation/withdrawal in the para position and hence were expected to vary the stability of the generated hydronitoxide radical that might act as an intermediary in inducing hemotoxic damage.

All phenylhydroxylamines in the present studies act as probable mediators of aniline-induced hemolytic anemia in rats; however, the interactions between phenylhydroxylamine and erythrocyte components which result ultimately in splenic sequestration of erythrocytes are poorly understood. It is known that phenylhydroxylamine is metabolized within erythrocytes and is a highly potent methemoglobin-forming agent in erythrocytes (Kiese, [5]). In as much as phenylhydroxylamine itself is a reducing agent, phenylhydroxylamine mediated hemoglobin oxidation is proposed to occur via a "coupled oxidation" with oxyhemoglobin in the erythrocyte, forming methemoglobin, nitrosobenzene and partially reduced oxygen species (e.g., peroxide and/or superoxide; Kiese [5]). Nitrosobenzene thus formed may be bound to deoxyhemoglobin or may be reduced to phenyl hydroxylamine by an NADPH dependent diaphorase, leading to the formation of a redox cycle producing many equivalents of methemoglobin for each equivalent of phenylhydroxylamine. The cycle is depleted eventually by side reactions involving phenylhydroxylamine or nitrosobenzene, or by reduction of phenylhydroxylamine to aniline within erythrocytes (Eyer [17], Kiese [5], Murayama [18]). Side reactions which may occur within erythrocytes include binding of phenylhydroxylamine to erythrocytic protein (Kiese and Traeger [19] and binding of both nitrosobenzene and phenylhydroxylamine to sulfhydryl groups in glutathione or protein (Boyland et al. [20], Eyer [17). The findings presented here support that several aniline metabolites of known structure mediate the hemolytic effect of aniline or any aniline-related compounds. Although it has been proposed previously that N-hydroxydapsone might mediate the hemolytic effects of dapsone (Glader and Conrad [21]), N-hydroxy metabolites have not otherwise been considered as mediators of the hemolytic effects of aniline, primaquine or other hemolytic drugs. Further experiments are required to determine whether aniline-induced hemolytic anemia is an appropriate model for hemolytic anemia induced by primaquine and other drugs 
which are aniline derivatives. Our data indicate that N-hydroxyl metabolites should be considered as possible mediators of chemical -induced hemolytic anemia, and thus are potential biomarkers for assessing chemical toxicity.

\section{Acknowledgements}

The authors thank Dr. David Jollow and Dr. David McMillan for the guidance and supervision of this study, Dr. John E. Otis for assistance in the synthesis of aniline analogs, Ms. Jennifer Schulte for the technical assistance and Dr. Sivapatham Paramasivam for the assistance in graphic presentation of data.

\section{References}

[1] Henderson, R.H., Biomarkers: The Genome \& the Individual, Charleston, SC, pp. 11, 1997.

[2] Harrison, J.H., Jr., Jollow, D.J., Role of aniline metabolites in anilineinduce hemolytic anemia. J Pharmacol Exp Ther 238:1045-1054, 1986.

[3] Harrison, J.H., Jr., Jollow, D.J., (Contribution of aniline metabolites to aniline-induced methemoglobinemia. Mol Pharmacol 32:423-431, 1987.

[4] Grossman, S.J., and Jollow, D.J., Role of Dapsone Hydroxylamine in Dapsone-Induced Hemolytic Anemia. The Journal of Pharmacology and Experimental Therapeutics 244(1): 118-125, 1987.

[5] Kiese, M., Methemoglobinemia: a comprehensive treatise. CEC Press, Cleveland, 1974.

[6] Evelyn, KA, Mallory, HT. Microdetermination of oxyhemoglobin, methemoglobin, and sulfhemoglobin in a single sample of blood. J Biol Chem 126:655-662, 1938.

[7] International Committee for Standardization in Hematology: Recommended methods for radioisotope red cell survival studies. Blood 38:378-386, 1971.

[8] von Jagow R, Kiese M, Renner G. Urinary excretion of $N$-hydroxy derivatives of some aromatic amines by rabbits, guinea pigs, and dogs. Biochem Pharmacol 15:1899-1910, 1966.

[9] Kiese, M., The biochemical production of ferrihemoglobin-forming derivatives from aromatic amines, and mechanisms of ferrihemoglobinemia formation. Pharmacol Rev 18:1091-1161, 1966.

[10] Eyer, P., Kampffmeyer H., Maister, H., Rösch-Oehme Biotransformation of nitrosobenzene, phenylhydroxylamine, and aniline in the isolated perfused rat liver. Xenobiotica 10:499-516, 1980.

[11] McLean, S., Starmer, G.A., Thomas, J., Methemoglobin formation by aromatic amines. J Pharm Pharmacol 21, 441, 1969.

[12] Kiese M. Oxydation von aniline zu nitrosobenzol in hunde. Nauyn Schmiedebergs Arch Pharmacol 235:360-364, 1969.

[13] Kiese, M., Renner, G., the isolation of p-chloronitrosobenzene from the blood of dogs injected with p-chloroaniline. Naunyn-Schmiedebergs Arch Exp Pathol Pharmakol 246, 163, 1963. 
[14] Williams JR, Challis FE Methylene blue as an antidote for aniline dye poisoning. Case report with confimatory experimental study. J Clin Med 19, 166, (1933).

[15] Hughes, J.P., Treon, J.F., erythrocytic inclusion bodies in the blood of chemical workers. Arch Ind Hyg Occup Med 10, 192, 1954.

[16] Faivre, M., Armand, J., Everux, JC., Duverneuil, G., Colin, C., Méthémoglobinémie toxique par des dérivés de paniline: parachloroaniline et paratoluidine (deux cas). Arch Mal Prof Méd trav 32, 575, 1971.

[17] Eyer, P., Reactions of nitrosobenzene with reduced glutathione. Chem.Biol. Interact. 24:227-239, 1979.

[18] Murayama M. The combining power of normal human hemoglobin for nitrosobenzene. J Biol Chem 235: 1024-1028, 1960.

[19] Kiese M, Traeger K. The fate if phenylhydroxylamine in human red cells. Naunyn-Schmiedeberg's Arch Pharmacol 292:59-66, 1976.

[20] Boyland, E, Mansi, D., and Nery, R. The reaction of phenylhydroxylamine and 2-naphthylhydroxylamine with thiols. J. Chem. Soc. Part I: 606-611,1962.

[21] Glader, B.E., and Conrad, M.E. Hemolysis by diphenylsulfones: Comparative effect of DDS and hydroxylamine-DDS. J. Lab. Clin. Med. 81:267-272, 1973. 